\title{
Quality of life outcomes after revision lumbar discectomy
}

\author{
Daniel Lubelski, BA, ${ }^{1-3}$ Nilgun Senol, MD, ${ }^{5}$ Michael P. Silverstein, MD, ${ }^{2,4}$ \\ Matthew D. Alvin, MBA, MA, ${ }^{2,6}$ Edward C. Benzel, MD, ${ }^{1-3}$ Thomas E. Mroz, MD, ${ }^{1-3}$ \\ and Richard Schlenk, MD ${ }^{1-3}$
}

\begin{abstract}
${ }^{1}$ Cleveland Clinic Lerner College of Medicine, ${ }^{2}$ Cleveland Clinic Center for Spine Health, and Departments of ${ }^{3}$ Neurological Surgery and ${ }^{4}$ Orthopaedic Surgery, Cleveland Clinic, Cleveland, Ohio; ${ }^{5}$ Department of Neurosurgery, Suleyman Demirel University School of Medicine, Isparta, Turkey; and ${ }^{6}$ Case Western Reserve University School of Medicine, Cleveland, Ohio
\end{abstract}

\begin{abstract}
OBJECT The authors investigated quality of life (QOL) outcomes after primary versus revision discectomy.
METHODS A retrospective review was performed for all patients who had undergone a primary or revision discectomy at the Cleveland Clinic Center for Spine Health from January 2008 through December 2011. Among patients in the revision cohort, they identified those who needed a second revision discectomy. Patient QOL measures were recorded before and after surgery. These measures included responses to the EQ-5D health questionnaire, Patient Health Questionnaire-9, Pain and Disability Questionnaire, and quality-adjusted life years (QALYs). Cohorts were compared by using independent-sample t-tests and Fisher exact tests for continuous and categorical variables, respectively. Multivariable logistic regression was performed to adjust for confounding.

RESULTS A total of 196 patients were identified (116 who underwent primary discectomy and 80 who underwent revision discectomy); average follow-up time was 150 days. There were no preoperative QOL differences between groups. Postoperatively, both groups improved significantly in all QOL measures. For QALYs, the primary cohort improved by 0.25 points $(p<0.001)$ and the revision cohort improved by 0.18 points $(p<0.001)$. QALYs improved for significantly more patients in the primary than in the revision cohort ( $76 \%$ vs $59 \%$, respectively; $p=0.02$ ), and improvement exceeded the minimum clinically important difference for more patients in the primary cohort ( $62 \%$ vs $45 \%$, respectively; $p=$ 0.03). Of the 80 patients who underwent revision discectomy, yet another recurrent herniation (third herniation) occurred in 14 (17.5\%). Of these, 4 patients (28.6\%) chose to undergo a second revision discectomy and the other $10(71.4 \%)$ underwent conservative management. For those who underwent a second revision discectomy, QOL worsened according to all questionnaire scores.
\end{abstract}

CONCLUSIONS QOL, pain and disability, and psychosocial outcomes improved after primary and revision discectomy, but the improvement diminished after revision discectomy.

http://thejns.org/doi/abs/10.3171/2014.10.SPINE14359

KEY WORDS revision discectomy; quality of life; QALY; Patient Health Questionnaire; Pain and Disability Questionnaire; lumbar

$\mathrm{L}$ UMBAR discectomy is the most commonly performed surgical spine procedure in the United States, with more than 300,000 procedures performed annually. ${ }^{1}$ Approximately $85 \%-90 \%$ of patients report favorable outcomes after discectomy. ${ }^{3,5,14}$ However, recurrent herniation as well as persistence and recurrence of symptoms after the initial procedure lead to revision discectomy for $5 \%-14 \%$ of patients. ${ }^{5,14,19,22}$

Although revision discectomy can alleviate recurring symptoms, it is also associated with additional risks for complications and adverse outcomes. ${ }^{16,19}$ However, complication rates after discectomy are low, and perioperative morbidity and hospital stay do not significantly differ after primary versus revision discectomy. ${ }^{17,16,19,20}$ Some studies have found clinical improvement to be similar after primary or revision discectomy; $;, 16,19,20$ however, other studies have reported that for a substantial number of patients, results are unfavorable after reoperation., ${ }^{4,6,10,12,16}$ Despite numerous studies on this topic, validated health status measures have not been used to effectively quantify quality of life (QOL) outcomes after primary compared with revision discectomy.

We therefore investigated QOL outcomes after primary and revision discectomy (i.e., 2-time herniation) by us-

ABBREVIATIONS MCID = minimum clinically important difference; PDQ = Pain and Disability Questionnaire; $P H Q-9=$ Patient Health Questionnaire-9; $Q A L Y=$ qualityadjusted life year; $\mathrm{QOL}=$ quality of life.

SUBMITTED April 7, 2014. ACCEPTED October 28, 2014.

INCLUDE WHEN CITING Published online December 5, 2014; DOI: 10.3171/2014.10.SPINE14359.

DISCLOSURE The authors report no conflict of interest concerning the materials or methods used in this study or the findings specified in this paper. 
ing validated health status measures: EQ-5D, the Patient Health Questionnaire-9 (PHQ-9), and the Pain and Disability Questionnaire (PDQ). Our hypothesis was that QOL would improve for all patients after primary and revision discectomy but that the improvements would be reduced after revision surgery.

\section{Methods}

We retrospectively reviewed records of all patients who had undergone a primary or revision discectomy at the Cleveland Clinic Center for Spine Health from January 2008 through December 2011. Institutional review board approval was obtained. The study design was not longitudinal; rather, the design comprised two separate populations of patients who, during this time frame, underwent either a primary or a revision discectomy. Patient and operative data were obtained from electronic medical records. We included in the study only those patients for whom both preoperative and postoperative QOL data were available. The QOL data were collected in conjunction with the institutional Knowledge Program, which is a patient-derived outcome assessment tool that is embedded in the electronic medical record. Before they were seen by the health care provider (pre- and postoperatively), all patients were given a portable tablet computer and asked to complete the questionnaire. The responses to these questions were then automatically transferred to the patients' medical record. Preoperative QOL data were collected an average of 27.8 days before surgery, and postoperative QOL data were collected an average of 150.6 days after surgery (mean [ \pm SD] 158.2 \pm 217.6 days for those in the primary discectomy group [primary cohort] and $139.4 \pm 132.4$ days for those in the revision discectomy group [revision cohort]). This difference between the groups was not significant. From the revision cohort, we subsequently identified a subset of patients who had experienced a third disc herniation (second recurrence) for which they then underwent either surgical or conservative management; we recorded posttreatment QOL data for this subset of patients.

QOL measures included responses to the EQ-5D, PHQ-9, and PDQ. For all measures except the EQ-5D, a decreased score represents improvement. The quality-adjusted life year (QALY) measure is derived from the EQ$5 \mathrm{D}$ and is calculated as follows: $\mathrm{QALY}=$ (years of life) $\times$ (utility value). For this equation, 1 year of perfect health is assigned the utility value of 1.0 and death is assigned a value of 0.0 ; anything between perfect health and death is assigned a value between 0.0 and 1.0. This utility value is based on the EQ-5D scores and converted to QALY values by using QOL standards for the United States. ${ }^{2,8,9,11}$ The PDQ uses an 11-point (0-10) scale to assess how pain affects the patient's ability to function in 15 categories. It is subdivided into functional and psychosocial components, which are combined to give a total score with a maximum of 150 points (higher scores indicate greater levels of pain/ disability). ${ }^{17}$ The PHQ-9 screens for depression according to the 9 criteria established by the Diagnostic and Statistical Manual of Mental Disorders, Fourth Edition, in which scores from 5 through 10 indicate minor depression and scores greater than 10 indicate major depression. ${ }^{18}$ The minimum clinically important differences (MCIDs) used for the EQ-5D, PDQ, and PHQ-9 scores at 1 year were $0.1,26$, and 5 , respectively. ${ }^{19-21}$

All data were analyzed using JMP 9.0 (SAS Institute Inc.). Descriptive statistics summarizing patient demographics are presented as means and SDs or counts with percentages, as appropriate. Paired t- and Wilcoxon signed-rank tests were used for parametric and nonparametric data, respectively, to ascertain significance in the preoperative to postoperative change in QOL score for each patient for the respective questionnaire. The primary and revision cohorts were compared with respect to numeric variables by using independent sample t-tests and with respect to categorical variables by using Fisher exact tests. Multivariable logistic regression was used to assess the association between success of surgery (as defined by QALYs exceeding the MCID) and revision versus primary surgery, while adjusting for potential confounders. Crude and adjusted odds ratios with $95 \%$ confidence intervals were calculated for the adjusted variables. All p values $\leq$ 0.05 were considered statistically significant.

\section{Results}

A total of 196 patients (116 primary discectomy, 80 revision discectomy) fit our inclusion criteria; 22 patients had been excluded because of incomplete questionnaire responses, but no differences were found between these patients and the study cohort. The mean age of patients in the primary cohort was 49 years and that of those in the revision cohort was 57 years $(\mathrm{p}=0.0004)$; the average follow-up time was 150.6 days (Table 1). The percentage of male patients in the revision cohort $(45 \%, 36 / 80)$ was significantly smaller than that in the primary cohort $(61 \%$, $71 / 116 ; p=0.03)$. No significant differences were observed between the cohorts in terms of race, marital status, smoking status, or follow-up time. Patients in the revision cohort were significantly more likely than those in the primary cohort to have had surgery performed at L3-4 (p = $0.01)$ and L4-5 (p=0.004) and at significantly more levels (1.5 vs 1.1 levels, $\mathrm{p}<0.0001)$. Preoperative QOL scores did not differ significantly between cohorts.

Postoperative scores for all QOL measures improved significantly over preoperative scores for patients in both cohorts (Table 2). Mean QALY scores for those in the primary cohort improved by 0.25 points (range $0.46-0.71$, $p$ $<0.001)$ and for those in the revision cohort by 0.18 points ( 0.40 to $0.58, \mathrm{p}<0.001)$. The change in QALY scores trended toward being significantly greater for patients in the primary than in the revision cohort $(\mathrm{p}=0.06)$, although both cohorts exceeded the MCID of 0.1. QALY scores improved for a significantly greater percentage of patients in the primary cohort ( $76 \%$ vs $59 \%, \mathrm{p}=0.02$ ), and improvement exceeded the MCID for a significantly greater percentage of these patients (62\% vs $45 \%, \mathrm{p}=0.03$ ). PHQ-9 depression scores did not differ significantly between the cohorts. Similarly, preoperative and postoperative changes in PDQ scores did not differ significantly (27.6 vs 23.8 for the primary vs revision cohort, respectively; $p=0.3$ ). Improvement exceeded the MCID for a significantly greater percentage of patients in the primary $(56 \%)$ than in the revision $(33 \%)$ cohort $(\mathrm{p}=0.04)$.

Because patients in both groups differed in several 
TABLE 1. Patient characteristics*

\begin{tabular}{|c|c|c|c|}
\hline \multirow[b]{2}{*}{ Variable } & \multicolumn{2}{|c|}{ Discectomy } & \multirow[b]{2}{*}{$\mathrm{p}$ Value } \\
\hline & Primary $(n=116)$ & Revision $(n=80)$ & \\
\hline Age (yrs) & $49.0 \pm 15.2$ & $56.8 \pm 14.4$ & $0.0004 \dagger$ \\
\hline Male & $71(61)$ & $36(45)$ & $0.03 \dagger$ \\
\hline Race & & & 0.7 \\
\hline White & $103(89)$ & $70(88)$ & \\
\hline Black & $5(4)$ & $7(9)$ & \\
\hline Marital status & & & 0.2 \\
\hline Married & $77(66)$ & $55(69)$ & \\
\hline Divorced & $14(12)$ & $6(8)$ & \\
\hline Single & $20(17)$ & $9(11)$ & \\
\hline Smoker & $25(22)$ & $17(21)$ & 0.99 \\
\hline \multicolumn{4}{|l|}{ Level operated on } \\
\hline L1-2 & $2(2)$ & $5(6)$ & 0.1 \\
\hline L2-3 & $5(4)$ & $10(13)$ & 0.05 \\
\hline L3-4 & $18(16)$ & $25(31)$ & $0.01 \dagger$ \\
\hline L4-5 & $47(41)$ & $50(63)$ & $0.004 \dagger$ \\
\hline L5-S1 & $55(47)$ & $27(34)$ & 0.08 \\
\hline Total spinal levels & $1.1 \pm 0.3$ & $1.5 \pm 0.8$ & $<0.0001 \dagger$ \\
\hline Preop QALY score & $0.46 \pm 0.2$ & $0.40 \pm 0.2$ & 0.1 \\
\hline Preop PHQ-9 score & $9.4 \pm 6.3$ & $8.9 \pm 6.8$ & 0.7 \\
\hline Preop PDQ score & $92.5 \pm 27.2$ & $100.0 \pm 31.4$ & 0.1 \\
\hline Follow-up duration & $158.2 \pm 217.6$ & $139.4 \pm 132.4$ & 0.5 \\
\hline
\end{tabular}

baseline characteristics (including demographic and operative characteristics), we performed a multivariable logistic regression to determine if QOL outcomes differed between primary and revision surgery, while adjusting for these baseline differences (Table 3). Variables controlled for included patient characteristics (e.g., age and sex) and operative characteristics (e.g., specific spinal levels and total levels operated on). When the baseline demographic and operative variables were adjusted for, primary surgery was found to be a significant independent predictor of successful postoperative outcome $(\mathrm{p}=0.001)$; odds of a successful outcome (QALY change exceeds the MCID) were 3.7 times greater for those who underwent primary surgery than for those who underwent revision surgery. Similarly, the preoperative QALY score was also a significant predictor of successful postoperative outcome.

Of the 80 patients who underwent a revision discectomy yet another recurrent herniation (third herniation) occurred in $14(17.5 \%)$. Of these, 4 patients $(28.6 \%)$ chose to undergo a second revision discectomy (i.e., a third discectomy surgery) and the other $10(71.4 \%)$ underwent conservative management. Whereas QOL scores improved for patients after the revision discectomy (albeit smaller improvements compared with the primary discectomy), QOL scores for all questionnaires worsened for all who underwent a second revision discectomy (Fig. 1). A comparison of QOL scores from first to second to third discectomy showed that QALY scores changed from $0.67 \pm 0.13$ to $0.73 \pm 0.12$ to $0.32 \pm 0.08$; PHQ-9 changed from $11 \pm 7.5$ to $3.3 \pm 2.6$ to $5 \pm 4.4$; and PDQ changed from $99 \pm 18.3$ to $68 \pm 35.7$ to $86.7 \pm 40.5$, respectively. Among those who experienced a recurrent herniation after the revision discectomy, QOL changes were significantly worse for those who underwent a third discectomy than for those who underwent conservative management of the third herniation. For those who underwent a third discectomy, QALY scores decreased from 0.64 after the second discectomy to 0.32 after the third discectomy (decrease of $0.32, \mathrm{p}=$ $0.02)$; in contrast, for those who underwent conservative management, QALY scores increased from 0.42 to 0.43 (increase of $0.01, \mathrm{p}=0.9$ ). This finding represented a significantly worse QOL outcome for those undergoing surgery for the third herniation $(\mathrm{p}=0.03)$.

\section{Discussion}

In the United States, it is a generally accepted practice to treat 1-time recurrent lumbar disc herniations with a revision discectomy. Similarly, 2-time recurrences are most often treated with a fusion procedure. There is a paucity of literature that has defined with validated measures the QOL for patients who undergo revision lumbar discectomy. Our intent with this study was to define these outcomes with the hypothesis that QOL would improve for all patients after primary and revision discectomy but that the improvements would be reduced after revision surgery.

Of the 196 patients who underwent primary and revision discectomy at our institution, most experienced 
TABLE 2. Preoperative to postoperative QOL changes

\begin{tabular}{|c|c|c|c|c|c|}
\hline \multirow[b]{2}{*}{ QOL Measure† } & \multicolumn{4}{|c|}{ Discectomy* $^{*}$} & \multirow[b]{2}{*}{$p$ Value (btwn groups) $\ddagger$} \\
\hline & \multicolumn{2}{|c|}{ Primary } & \multicolumn{2}{|c|}{ Revision } & \\
\hline QALY score & & & & & \\
\hline Preop & 0.46 & \multirow{3}{*}{$p<0.001 \S$} & 0.40 & \multirow{3}{*}{$p<0.001 \S$} & \multirow{3}{*}{0.06} \\
\hline Postop & 0.71 & & 0.58 & & \\
\hline$\Delta$ & 0.25 & & 0.18 & & \\
\hline Any improvement & \multicolumn{2}{|c|}{$77(76)$} & \multicolumn{2}{|c|}{$42(59)$} & $0.02 \ddagger$ \\
\hline Improved > MCID & \multicolumn{2}{|c|}{$63(62)$} & \multicolumn{2}{|c|}{$32(45)$} & $0.03 \ddagger$ \\
\hline \multicolumn{6}{|l|}{ PHQ-9 score } \\
\hline Preop & 10.1 & \multirow{3}{*}{$p=0.007 \S$} & 10.2 & \multirow{3}{*}{$p=0.03 \S$} & \multirow{3}{*}{0.7} \\
\hline Postop & 5.8 & & 5.9 & & \\
\hline$\Delta$ & 4.3 & & 4.3 & & \\
\hline Any improvement & \multicolumn{2}{|c|}{$31(69)$} & \multicolumn{2}{|c|}{$21(60)$} & 0.5 \\
\hline Improved > MCID & \multicolumn{2}{|c|}{$16(36)$} & \multicolumn{2}{|c|}{$8(23)$} & 0.3 \\
\hline \multicolumn{6}{|l|}{ PDQ score } \\
\hline Preop & 90.7 & \multirow{3}{*}{$p<0.001 \S$} & 103.5 & \multirow{3}{*}{$p=0.009 \S$} & \multirow{3}{*}{0.3} \\
\hline Postop & 63.1 & & 79.8 & & \\
\hline$\Delta$ & 27.6 & & 23.8 & & \\
\hline Any improvement & \multicolumn{2}{|c|}{$50(82)$} & \multicolumn{2}{|c|}{$27(75)$} & 0.4 \\
\hline Improved > MCID & \multicolumn{2}{|c|}{$34(56)$} & \multicolumn{2}{|c|}{$12(33)$} & $0.04 \ddagger$ \\
\hline
\end{tabular}

\footnotetext{
* Because not all questionnaires were completed by every patient at every visit, percentages are based on the total number of patients for whom both preoperative and postoperative scores for the given questionnaire were available.

$\dagger \Delta$ represents the change in score; improved $>$ MCID represents the number and percentage of patients for whom improvement exceeded the respective MCID threshold. Categorical variables are presented as number (\%).

$\ddagger p$ values were used to compare changes between the primary and revision discectomy groups and were calculated by using independent t-tests.

$\S$ Significant $(p \leq 0.05)$. Paired t-tests and Wilcoxon signed-rank tests were used for parametric and nonparametric data, respectively, when analyzing preoperative to postoperative changes.
}

preoperative to postoperative improvement in all QOL measures. For many, these improvements were not only statistically significant, but they were also clinically significant (as indicated by exceeding the MCID). Although improvement was experienced in all groups, EQ-5D QALY scores improved most for those in the primary co-

TABLE 3. Multivariable logistic regression analysis of successful surgery*

\begin{tabular}{lcl}
\hline \multicolumn{1}{c}{ Variable } & Odds Ratio $(95 \% \mathrm{Cl})$ & $\mathrm{p} \mathrm{Value}$ \\
\hline Primary surgery & $3.7(1.7-8.4)$ & $0.001 \dagger$ \\
\hline Patient age & $1.0(0.97-1.03)$ & 0.5 \\
\hline Patient sex (M/F) & $1.4(0.7-3.0)$ & 0.3 \\
\hline Level of surgery & & \\
\hline L2-3 & $0.3(0.02-7.7)$ & 0.5 \\
\hline L3-4 & $1.9(0.2-21.4)$ & 0.6 \\
\hline L4-5 & $0.9(0.1-9.9)$ & 0.9 \\
\hline L5-S1 & $1.5(0.2-16.4)$ & 0.7 \\
\hline Total op levels & $1.3(0.1-10.3)$ & 0.8 \\
\hline Preop QALY score & $139(20.4-1145)$ & $<0.0001 \dagger$ \\
\hline
\end{tabular}

* Successful surgery defined as patients for whom change in preoperative to postoperative QALY score exceeded the MCID of 0.1. Variables to include in the regression model were based on whether they had a trend toward significance at baseline by univariate analysis, as well as clinical relevance. $\dagger$ Significant $(p \leq 0.05)$. hort. Furthermore, the percentage of patients who experienced any improvement and the percentage of those who experienced improvement greater than the MCID was significantly greater for the primary versus revision cohort. This difference between primary and revision cohorts was seen despite preoperative QOL similarity for both groups.

Although previous studies have not used the validated EQ-5D, PHQ-9, and PDQ measures to compare primary versus revision discectomy QOL outcomes, other studies have compared the surgical outcomes between these groups. Most of these previous studies did not find significantly different outcomes. Suk et al. ${ }^{19}$ retrospectively reviewed data collected from 28 patients who had undergone a revision discectomy after a primary discectomy at their institution. The authors found no significant difference in clinical improvement when comparing revision and primary surgeries, as interpreted from their review of the medical record.

Cinotti et al. ${ }^{3}$ prospectively compared outcomes obtained in 26 patients who had undergone revision discectomy with outcomes in 50 control patients who had undergone a primary discectomy. They found no significant difference between groups; postoperative satisfaction was reported by $85 \%$ of those in the revision group and $88 \%$ in the control primary discectomy group. They also found no difference between groups in ability to perform daily activities. 

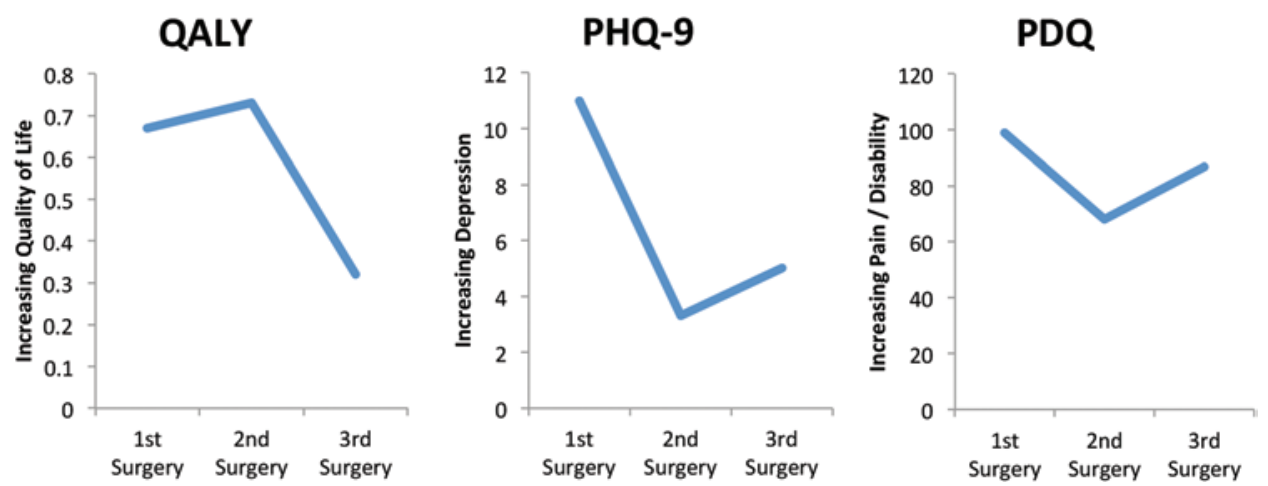

FIG. 1. Average QOL outcome measure score changes from first to second (revision) to third (second revision) discectomy. Figure is available in color online only.

In a retrospective matched cohort study, Papadopoulos et al. ${ }^{16}$ reviewed the records of 27 patients who had undergone a revision discectomy and 30 patients who had undergone a primary discectomy. The authors contacted the patients by telephone and asked them to complete the Musculoskeletal Outcomes Data Evaluation and Management System outcomes measure. This questionnaire assesses a range of factors including pain, function, neurological deficit, and psychological factors. The authors found no significant difference in satisfaction after surgery or in any of the function outcomes; they did, however, find that leg numbness was significantly more frequent and severe and that low-back/buttock pain was significantly more frequent for those in the revision cohort than for those in the primary discectomy group. In our study, pain/disability outcomes were significantly worse and QOL improvements were significantly lower for patients who had undergone revision discectomy than for those who had undergone a primary discectomy. One explanation for the differences between the outcomes of our study and those of the Papadopoulos et al. study might be the use of different validated QOL outcome measures. Another explanation might be that the sample size for the Papadopoulos et al. study was small and may have been underpowered to detect more subtle differences between groups.

The findings from our study demonstrating inferior postoperative outcomes for those undergoing revision discectomy relative to primary discectomy are supported by previous studies in the spine surgery literature. Previous reports have indicated that patients undergoing a primary discectomy have a $76 \%-95 \%$ chance of satisfactory results as measured by pain and functional measures, whereas only $59 \%-81 \%$ of those undergoing a revision discectomy will achieve the same results. ${ }^{6,15,18,21,23}$

Although our findings suggest that QOL improvements are less for those undergoing revision discectomy than for those undergoing primary discectomy, the improvements experienced by revision discectomy patients nonetheless often exceed the MCID threshold. In contrast, QOL outcomes tend to be relatively poor for patients who experience another recurrent (third) herniation after revision discectomy. In our cohort, a third herniation at the same level as the original herniation (index level) was experienced by a fairly large number of patients (17.5\%) after the revision procedure. Scores for all questionnaires (EQ-5D,
PHQ-9, and PDQ) tended to improve after the first revision procedure but worsen after the patient experienced another herniation. This latter worsening, however, was largely a function of undergoing a second revision procedure. In our study, 4 (28.6\%) of 14 patients chose to undergo a third discectomy (second revision discectomy), and the rest received nonsurgical treatment. Scores worsened significantly for those who underwent surgery (QALY scores decreased by 0.32 ), whereas they virtually did not change for those who received conservative management (increase of 0.01).

These data have several major implications. Patients who choose to undergo a primary discectomy or revision discectomy will probably experience postoperative improvement in their QOL scores. Patients, however, should be made aware of the likelihood that their postoperative QOL improvement after a revision discectomy will not be as good as the postoperative improvement after the primary discectomy. Approximately $18 \%$ of patients will experience yet another herniation after the revision discectomy; for these patients, QOL outcomes will be better if they undergo conservative management than if they undergo a third discectomy at the same level as the original herniation. However, it should be noted that this preliminary conclusion is based on a small sample size and thus needs further validation. On a healthcare economics level, we can use these QOL outcomes to evaluate cost-effectiveness of performing revision versus primary discectomies.

Ambrossi et al. ${ }^{1}$ performed a retrospective health care cost analysis of patients who underwent either revision surgery or conservative therapy for symptomatic samelevel recurrent disc herniations. The authors did not assess validated QOL outcomes. They found that the mean cost of conservative management was $\$ 2315$, whereas the mean cost of revision discectomy was $\$ 39,836$. In another study, Parker et al. ${ }^{17}$ found that the mean cost per patient for primary discectomy surgery was similar to that for revision discectomy, $\$ 42,554$. In a randomized study, Malter et $a .^{13}$ analyzed insurance data from 372 patients who had undergone discectomy and found that the mean cost for these patients was $\$ 13,390$.

Using the aforementioned costs reported in the literature as well as the QALY results obtained in our study enabled us to perform a preliminary cost-effectiveness analysis. This analysis can be used to estimate the value of the revision versus the primary discectomy. The cost effec- 
tiveness can be reported as the cost of the surgery divided by the gain in QALY from the respective surgery. On the basis of our analysis, the cost/QALY gained for primary discectomy would be $\$ 53,560-\$ 170,216$, whereas that for revision discectomy would be $\$ 74,389-\$ 221,311$ (depending on the actual cost of surgery). According to results of this rough analysis, although the procedure may not fall below the \$50,000-\$100,000/QALY-gained threshold, ${ }^{11}$ the revision discectomy is nonetheless less cost effective by $\$ 20,829-\$ 51,095 /$ QALY gained.

Our study has several limitations that must be considered when interpreting the results. It was a retrospective study with only a limited sample size and a single postoperative time point. Follow-up times varied, although they were not significantly associated with outcome and therefore did not confound the results. Future prospective studies are needed for evaluation of the change in postoperative QOL over time. There were also differences between the cohorts that could potentially confound the results; we attempted to account for these by using multivariable logistic regression analysis. To our knowledge, however, ours is the first study to investigate the difference in QALY, PHQ-9, and PDQ outcomes for patients undergoing revision versus primary discectomy. Our study included the largest number of patients of any study reported in the literature and used a unique combination of validated QOL questionnaires, which together provide psychosocial and QOL outcome information about this prevalent spine surgery population.

\section{Conclusions}

Our study demonstrated that QOL, pain and disability, and psychosocial outcomes improve after both primary and revision discectomy but that the improvement is diminished after the revision discectomy. Moreover, because the QOL gained decreases after revision discectomy and the costs of primary and revision discectomies are similar, the cost effectiveness of revision discectomy is less than that of primary discectomy. These results can enable clinicians and patients to better understand the postoperative QOL outcomes associated with primary and revision discectomy and thereby be more informed about treatment options. To validate our findings, prospective studies using these or equivalent QOL instruments are needed.

\section{References}

1. Ambrossi GLG, McGirt MJ, Sciubba DM, Witham TF, Wolinsky JP, Gokaslan ZL, et al: Recurrent lumbar disc herniation after single-level lumbar discectomy: incidence and health care cost analysis. Neurosurgery 65:574-578, 2009

2. Badia X, Diaz-Prieto A, Gorriz MT, Herdman M, Torrado H, Farrero E, et al: Using the EuroQol-5D to measure changes in quality of life 12 months after discharge from an intensive care unit. Intensive Care Med 27:1901-1907, 2001

3. Cinotti G, Roysam GS, Eisenstein SM, Postacchini F: Ipsilateral recurrent lumbar disc herniation. A prospective, controlled study. J Bone Joint Surg Br 80:825-832, 1998

4. Connolly ES: Surgery for recurrent lumbar disc herniation. Clin Neurosurg 39:211-216, 1992

5. Daneyemez M, Sali A, Kahraman S, Beduk A, Seber N: Outcome analyses in 1072 surgically treated lumbar disc herniations. Minim Invasive Neurosurg 42:63-68, 1999
6. Ebeling U, Kalbarcyk H, Reulen HJ: Microsurgical reoperation following lumbar disc surgery. Timing, surgical findings, and outcome in 92 patients. J Neurosurg 70:397-404, 1989

7. Erbayraktar S, Acar F, Tekinsoy B, Acar U, Güner EM: Outcome analysis of reoperations after lumbar discectomies: a report of 22 patients. Kobe J Med Sci 48:33-41, 2002

8. Jansson KA, Németh G, Granath F, Jönsson B, Blomqvist $P$ : Health-related quality of life in patients before and after surgery for a herniated lumbar disc. J Bone Joint Surg Br 87:959-964, 2005

9. Johnson JA, Coons SJ, Ergo A, Szava-Kovats G: Valuation of EuroQOL (EQ-5D) health states in an adult US sample. Pharmacoeconomics 13:421-433, 1998

10. Jönsson B, Strömqvist B: Repeat decompression of lumbar nerve roots. A prospective two-year evaluation. J Bone Joint Surg Br 75:894-897, 1993

11. Kepler CK, Wilkinson SM, Radcliff KE, Vaccaro AR, Anderson DG, Hilibrand AS, et al: Cost-utility analysis in spine care: a systematic review. Spine J 12:676-690, 2012

12. Law JD, Lehman RA, Kirsch WM: Reoperation after lumbar intervertebral disc surgery. J Neurosurg 48:259-263, 1978

13. Malter AD, Larson EB, Urban N, Deyo RA: Cost-effectiveness of lumbar discectomy for the treatment of herniated intervertebral disc. Spine (Phila Pa 1976) 21:1048-1055, 1996

14. Moore AJ, Chilton JD, Uttley D: Long-term results of microlumbar discectomy. Br J Neurosurg 8:319-326, 1994

15. O'Sullivan MG, Connolly AE, Buckley TF: Recurrent lumbar disc protrusion. Br J Neurosurg 4:319-325, 1990

16. Papadopoulos EC, Girardi FP, Sandhu HS, Sama AA, Parvataneni HK, O'Leary PF, et al: Outcome of revision discectomies following recurrent lumbar disc herniation. Spine (Phila Pa 1976) 31:1473-1476, 2006

17. Parker SL, Xu R, McGirt MJ, Witham TF, Long DM, Bydon A: Long-term back pain after a single-level discectomy for radiculopathy: incidence and health care cost analysis. J Neurosurg Spine 12:178-182, 2010

18. Patel N, Pople IK, Cummins BH: Revisional lumbar microdiscectomy: an analysis of operative findings and clinical outcome. Br J Neurosurg 9:733-737, 1995

19. Suk KS, Lee HM, Moon SH, Kim NH: Recurrent lumbar disc herniation: results of operative management. Spine (Phila Pa 1976) 26:672-676, 2001

20. Swartz KR, Trost GR: Recurrent lumbar disc herniation. Neurosurg Focus 15(3):E10, 2003

21. Thomas AM, Afshar F: The microsurgical treatment of lumbar disc protrusion. Follow-up of 60 cases. J Bone Joint Surg Br 69:696-698, 1987

22. Williams RW: Microlumbar discectomy. A 12-year statistical review. Spine (Phila Pa 1976) 11:851-852, 1986

23. Wilson DH, Kenning J: Microsurgical lumbar discectomy: preliminary report of 83 consecutive cases. Neurosurgery 4:137-140, 1979

\section{Author Contributions}

Conception and design: Schlenk, Lubelski. Acquisition of data: Lubelski, Senol, Silverstein. Analysis and interpretation of data: Schlenk, Lubelski, Alvin, Benzel, Mroz. Drafting the article: Lubelski, Alvin. Critically revising the article: Schlenk, Lubelski, Senol, Silverstein, Benzel, Mroz. Reviewed submitted version of manuscript: all authors. Statistical analysis: Lubelski.

\section{Correspondence}

Richard Schlenk, Center for Spine Health, Department of Neurological Surgery, The Cleveland Clinic, 9500 Euclid Ave., S-40, Cleveland, OH 44195. email: schlenr@ccf.org. 